\title{
Study of dipolar fluid inclusions in charged random matrices
}

\author{
M. J. Fernaud, E. Lomba, ${ }^{\text {a) }}$ and C. Martín \\ Instituto de Química Física Rocasolano (CSIC), Serrano 119, E-28006 Madrid, Spain \\ D. Levesque and J.-J. Weis \\ Laboratoire de Physique Théorique, UMR 8627, Bâtiment 210, Université Paris-Sud, \\ 91405 Orsay Cedex, France
}

(Received 13 March 2003; accepted 31 March 2003)

\begin{abstract}
Structural, thermodynamic, and dielectric properties of a dipolar fluid confined in a charged random matrix are studied by means of grand canonical Monte Carlo simulation and replica OrnsteinZernike integral equations in the hypernetted chain approximation. The fluid is modeled by a system of dipolar hard spheres. Two matrix topologies are considered: a frozen restricted primitive model matrix and a frozen hard sphere fluid with randomly distributed negative and positive charges. Both models lead to similar results in most cases, with significant deviations from the behavior of the corresponding equilibrated mixtures. The dielectric behavior is particularly interesting, since the effect of partial quenching on the equilibrated mixture recovers the electrostatics of the pure dipolar fluid but with the presence of Coulomb tails in the dipole-dipole total correlations. Differences between the two matrix models arise more vividly in the low density regime, in which the matrix with randomly distributed charges tends to enhance dipole association around the matrix particles. The integral equation results are in relatively good agreement with the computer simulation estimates. (c) 2003 American Institute of Physics. [DOI: 10.1063/1.1576377]
\end{abstract}

\section{INTRODUCTION}

Fluid inclusion in random porous matrices is a problem of interest both from the scientific and technological standpoints, due to its implications in purification processes, catalysis, and eventually in what is nowadays considered nanotechnology. Simple models for these systems that can be tackled with the standard tools of statistical mechanics have been devised during the last decade with a varying degree of complexity, ranging from the simple hard sphere fluid in a hard sphere matrix system, ${ }^{1}$ to models incorporating dispersion forces, ${ }^{2}$ molecular fluids, ${ }^{3,4}$ associating fluids, ${ }^{5,6}$ mixtures, ${ }^{7}$ etc. As to the nature of the matrix, these treatments are all based on the partial quenching of the positions of one of the components in a multicomponent mixture. In addition to the simple matrix formed from quenched hard sphere configurations and randomly positioned hard spheres, ${ }^{8}$ more realistic structures can also be generated using templated materials, ${ }^{9}$ even constituted from polydisperse mixtures. ${ }^{10}$

Particular attention has also been paid to charged matrix systems. ${ }^{11,12}$ Charges play a central role in adsorption processes involved in liquid chromatography and consequently the modeling of a charged random matrix goes beyond the purely academic interest. As found in Refs. 11 and 12 the partial quenching of the matrix particles modifies the screening behavior of the fluid-fluid correlations when the adsorbed particles are also charged. Large effects should also be expected for dipolar fluid inclusions in charged matrices. Actually, it has been found in many instances that the properties of the partially quenched system differ very little from

${ }^{\text {a)} E l e c t r o n i c ~ m a i l: ~ e . l o m b a @ i q f r . c s i c . e s ~}$ those of the corresponding fully equilibrated mixture. ${ }^{13}$ This will certainly not hold for the charged matrix systems since in this case the freezing of the matrix positions hinders the ionic reorganization required to attain the screening that one observes in equilibrated mixtures of electrical particles. Moreover, the problem of a dipolar fluid adsorbed in random matrices has recently been studied in detail, in particular the behavior of the dielectric constant. ${ }^{14,15}$ This system represents an adequate starting point and reference to analyze the effect of the presence of charges in the matrix on the dielectric and screening behavior, which constitutes the main purpose of this work.

To this aim, we have resorted here to the use of grand canonical Monte Carlo simulations (GCMC), and the replica Ornstein-Zernike (ROZ) integral equation, which since the pioneering work of Given and Stell ${ }^{16}$ has been successfully applied to an increasingly large number of models of partly quenched mixtures.

In this paper we will consider the matrix constituted by quenched charged hard spheres, whose interaction is given by

$$
\beta_{0} u_{00}^{\alpha \gamma}(r)=\left\{\begin{array}{l}
\infty \quad \text { if } r<\sigma_{00}^{\alpha \gamma}, \\
\frac{\beta_{0} Z_{\alpha} Z_{\gamma} e^{2}}{r} \text { if } r \geqslant \sigma_{00}^{\alpha \gamma},
\end{array}\right.
$$

where $e$ is the electron charge, $Z_{\nu}$ is the charge of the ions of type $\nu, \beta_{0}=1 / k_{B} T_{0}$ is the inverse temperature at which the charged hard spheres have been quenched, and $\sigma_{00}^{\alpha \gamma}$ is the overlap diameter between two matrix particles of types $\alpha$ and $\gamma$. Here we will limit ourselves to the simplest case of $\left|Z_{\alpha}\right|=\left|Z_{\beta}\right|=Z=1$, and $\sigma_{00}^{++}=\sigma_{00}^{--}=\sigma_{00}^{+-}=\sigma_{00}$. As to the matrix-fluid interaction it will be given by 


$$
\beta u_{ \pm d}\left(r, \omega_{1}\right)=\left\{\begin{array}{l}
\infty \quad \text { if } r<\sigma_{0 d}, \\
-\frac{\beta Z_{ \pm} \mu e}{r^{2}} \cos \theta_{r 1} \quad \text { if } r \geqslant \sigma_{0 d},
\end{array}\right.
$$

where $\sigma_{0 d}=\left(\sigma_{00}+\sigma_{d d}\right) / 2, \sigma_{d d}$ is the hard sphere diameter of the dipolar particles, $\beta=1 / k_{B} T$ the inverse temperature of the dipoles in the matrix, $\mu$ is the dipole moment, and $\theta_{r 1}$ is the angle formed by the dipole moment and the vector $\mathbf{r}$ joining the centers of particles 0 and 1 . Finally, the fluidfluid interaction is given by

$$
\begin{aligned}
& \beta u_{d d}\left(r, \omega_{1}, \omega_{2}\right) \\
& =\left\{\begin{array}{l}
\infty \quad \text { if } r<\sigma_{d d}, \\
\frac{-\beta \mu^{2}}{r^{3}}\left(3\left(\hat{s}_{1} \cdot \hat{r}\right)\left(\hat{s}_{2} \cdot \hat{r}\right)-\hat{s}_{1} \cdot \hat{s}_{2}\right) \quad \text { if } r \geqslant \sigma_{d d},
\end{array}\right.
\end{aligned}
$$

where $\hat{s}_{i}$ and $\hat{r}$ are unit vectors describing the orientation of the dipole moment on particle $i$ and the orientation of the interparticle axis, respectively. Now, not only the spatial distribution of the matrix particles will be important, but the charge distribution as well. Therefore here we will consider two different matrix configurations obtained by quenching the ionic particles in an ion-dipole mixture with reduced (inverse) temperatures $\beta_{0} e^{2} / \sigma_{00}=1$ and $\beta_{0} e^{2} / \sigma_{00}=0.005$. In the latter case the charge distribution in the matrix is practically random and the spatial distribution is identical to that of the hard sphere fluid.

We will see that, as expected, the presence of charges in the matrix strongly modifies the long-range (i.e., screening) behavior of the fluid correlation functions. It will be shown that the ROZ predictions are in reasonable agreement with the GCMC results for the structure, thermodynamic and dielectric properties. For the sake of comparison, calculations for the corresponding fully equilibrated system-i.e., an electrolyte with explicit solvent-have also been carried out in the hypernetted chain (HNC) approximation, which is known to be accurate for the thermodynamic states here considered. This comparison will illustrate more clearly the effects of confinement on the dipolar fluid properties.

The rest of the paper is organized as follows: Section II is devoted to a brief presentation of the ROZ integral equation for this system, including the expressions for the evaluation of thermodynamic and dielectric properties. In Sec. III we will analyze the long-range behavior of the correlation functions. Section IV contains a brief summary of the specific details of the GCMC simulations, and finally in Sec. V we present our most significant results.

\section{THE ROZ INTEGRAL EQUATION APPROACH}

The ROZ formalism to treat the ion-dipole mixture is essentially the same used for the diatomic fluid ${ }^{3}$ or the dipolar fluid ${ }^{14}$ adsorbed in random matrices, with the particularity that now the matrix incorporates two species. This has been also considered in Refs. 11 and 12 for the primitive model electrolyte adsorbed in an electroneutral charged matrix. Here we only have to add the orientational dependence of the fluid-fluid and fluid-matrix correlations as done in Ref. 3. In this way one gets the following set of matrix equations:

$$
\begin{aligned}
\boldsymbol{\Gamma}^{d-}= & -\mathbf{C}^{d-}+\mathbf{G}_{m}\left[S_{-} \mathbf{C}^{d-}+\rho_{+} \widetilde{h}_{+-} \mathbf{C}^{d+}\right], \\
\boldsymbol{\Gamma}^{d+}= & -\mathbf{C}^{d+}+\mathbf{G}_{m}\left[S_{+} \mathbf{C}^{d+}+\rho_{-} \widetilde{h}_{-+} \mathbf{C}^{d-}\right], \\
\boldsymbol{\Gamma}_{m}^{d d}= & -\mathbf{C}_{m}^{d d}+\mathbf{G}_{m}\left[\mathbf{C}_{m}^{d d}+(-1)^{m}\left(\rho_{-} S_{-} \mathbf{C}^{d-} \mathbf{C}^{-d} \mathbf{G}_{m}\right.\right. \\
& +2 \rho_{-} \rho_{+} \widetilde{h}_{-+} \mathbf{C}^{d-} \mathbf{C}^{+d} \mathbf{G}_{m}+\rho_{+} S_{+} \mathbf{C}^{d+} \mathbf{C}^{+d} \mathbf{G}_{m} \\
& \left.\left.+\rho_{d} \mathbf{C}_{m}^{d d^{\prime}} \mathbf{G}_{m} \mathbf{C}_{m}^{c}\right]\right), \\
\boldsymbol{\Gamma}_{m}^{c}= & (-1)^{m} \rho_{d} \mathbf{G}_{m} \mathbf{C}_{m}^{c 2}
\end{aligned}
$$

with

$$
\begin{aligned}
& \mathbf{G}_{m}=\left[\mathbf{I}-(-1)^{m} \rho_{d} \mathbf{C}_{m}^{c}\right]^{-1}, \\
& S_{-}=1+\rho_{-} \widetilde{h}_{--}, \\
& S_{+}=1+\rho_{+} \tilde{h}_{++},
\end{aligned}
$$

where $\mathbf{I}$ is the identity matrix, $\tilde{h}_{\alpha \beta}$ are the Fourier transforms of the matrix-matrix total correlation function, and the elements of the remaining matrices are the following: $\left[\boldsymbol{\Gamma}_{m}^{d d}\right]_{k l}$ $=\widetilde{h}_{k l m}^{d d}-\tilde{c}_{k l m}^{d d},\left[\mathbf{C}_{m}^{d d}\right]_{k l}=\tilde{c}_{k l m}^{d d},\left[\boldsymbol{\Gamma}^{d \pm}\right]_{k}=\widetilde{h}_{k 00}^{d \pm}-\tilde{c}_{k 00}^{d \pm}$ and similarly for $\mathbf{C}^{d \pm}$. Here $\tilde{c}^{d d}, \tilde{c}^{d \pm}$ denote the Fourier transform of the dipole-dipole and dipole-charge direct correlation functions, respectively, $\rho_{d}$ is the dipole number density, and $\rho_{ \pm}$ the matrix anion or cation densities, and $\widetilde{h}^{d d}, \widetilde{h}^{d \pm}$ are the dipole-dipole and dipole-charge (i.e., fluid-matrix) total correlation functions. The subindices $(\mathrm{klm})$ identify the corresponding coefficients of the expansion of the correlation functions in spherical harmonics. ${ }^{3}$ Finally, $\left[\mathbf{C}_{m}^{d d^{\prime}}\right]_{k l}=\widetilde{c}_{k l m}^{d d}$ $=\tilde{c}_{k l m}^{12}$ is the Fourier transform of the replica-replica direct correlation function (blocking function), and the connected function is defined as usual by $c^{c}=c^{d d}-c^{d d^{\prime}}$, and similarly for $h^{c}$. Let us recall that the replicated particles are the dipolar hard spheres, i.e., the annealed fluid in the partly quenched mixture.

These equations must be complemented with a closure relation in $r$ space, for which in this work we have chosen the HNC approximation. This equation is known to give reasonable results for ionic fluids, and consequently we can expect a similar behavior here. In the present instance the HNC can be written as

$$
\begin{aligned}
h_{l 00}^{d \pm}\left(r_{12}\right)= & \left\langle\operatorname { e x p } \left[-\beta u^{d \pm}\left(r_{12}, \omega_{1}\right)+h^{d \pm}\left(r_{12}, \omega_{1}\right)\right.\right. \\
& \left.-c^{d \pm}\left(r_{12}, \omega_{1}\right)\right]|l 00\rangle-\delta_{l 0} \\
h_{l_{1} l_{2} m}^{d d}\left(r_{12}\right)= & \left\langle\operatorname { e x p } \left[-\beta u^{d d}\left(r_{12}, \omega_{1}, \omega_{2}\right)+h^{d d}\left(r_{12}, \omega_{1}, \omega_{2}\right)\right.\right. \\
& \left.-c^{d d}\left(r_{12}, \omega_{1}, \omega_{2}\right)\right]\left|l_{1} l_{2} m\right\rangle-\delta_{l_{1} l_{2} m, 000} \\
h_{l_{1} l_{2} m}^{d d^{\prime}}\left(r_{12}\right)= & \left\langle\operatorname { e x p } \left[ h^{d d^{\prime}}\left(r_{12}, \omega_{1}, \omega_{2}\right)\right.\right. \\
& \left.-c^{d d^{\prime}}\left(r_{12}, \omega_{1}, \omega_{2}\right)\right]\left|l_{1} l_{2} m\right\rangle-\delta_{l_{1} l_{2} m, 000}
\end{aligned}
$$

where $\left\langle\ldots \mid l_{1} l_{2} m\right\rangle$ denotes the projection onto the spherical harmonic basis function $Y_{l_{1} m}\left(\omega_{1}\right) Y_{l_{2} \bar{m}}\left(\omega_{2}\right)$. Additionally, an 
HNC equation for the matrix decouples completely and can be solved by the standard procedures devised for primitive model electrolytes. ${ }^{17}$

\section{A. Thermodynamic properties}

Most thermodynamic properties can be expressed in terms of the correlation functions. We need not go into details of the derivations that can be found elsewhere, ${ }^{3,18}$ so here we just present the expressions that enable their calculation.

\section{Excess internal energy}

Using the replica trick, one gets for the net fluid-fluid and fluid-matrix contribution to the internal energy

$$
\begin{aligned}
\frac{\beta U_{1}^{\mathrm{ex}}}{V}= & \rho_{d} \rho_{+} \int u_{100}^{d+}(r) h_{100}^{d+}(r) d \mathbf{r} \\
& +\rho_{d} \rho_{-} \int u_{100}^{d-}(r) h_{100}^{d-}(r) d \mathbf{r} \\
& +\frac{\rho_{d}^{2}}{2} \int\left(u_{110}^{d d}(r) h_{110}^{d d}(r)+2 \int u_{111}^{d d}(r) h_{111}^{d d}(r)\right) d \mathbf{r},
\end{aligned}
$$

where the coefficients of the dipole-dipole and ion-dipole interaction are

$$
\begin{aligned}
& u_{110}^{d d}(r)=-\frac{2}{3} \frac{\mu^{2}}{r^{3}}, \\
& u_{111}^{d d}(r)=-\frac{1}{3} \frac{\mu^{2}}{r^{3}}, \\
& u_{100}^{d \pm}=\frac{Z_{ \pm} \mu}{\sqrt{3} r^{2}} .
\end{aligned}
$$

The first two terms in Eq. (6) give the dipole-matrix (and matrix-dipole) contribution, $\beta U_{10}^{\mathrm{ex}} / V$, and the last term the dipole-dipole contribution, $\beta U_{11}^{\mathrm{ex}} / V$.

\section{Dipolar fluid excess chemical potential}

A closed expression for the evaluation of this quantity can be easily derived in the HNC approximation using Lee's star function technique ${ }^{19}$ and the replica trick, ${ }^{14}$ leading to

$$
\begin{aligned}
\beta \mu_{1}^{\mathrm{ex}}= & -\rho_{+} \widetilde{c}_{000}^{d+}(0)-\rho_{-} \tilde{c}_{000}^{d-}(0)-\rho_{d} \widetilde{c}_{000}^{d d}(0)+\rho_{d} \widetilde{c}_{000}^{d d^{\prime}}(0)+\rho_{+} \sum_{l_{1} \neq 1} \int h_{0 l_{1} 0}^{+d}(r) \gamma_{0 l_{1} 0}^{+d}(r) d \mathbf{r}+\rho_{+} \int h_{010}^{+d}(r) \gamma_{010}^{+d *}(r) d \mathbf{r} \\
& +\rho_{-}\left[\sum_{l_{1} \neq 1} \int h_{0 l_{1} 0}^{-d}(r) \gamma_{0 l_{1} 0}^{-d}(r) d \mathbf{r}+\int h_{010}^{-d}(r) \gamma^{-d *}(r) d \mathbf{r}\right]-\frac{\rho_{-} Z_{+} e \mu}{\sqrt{3}} \int h_{010}^{+d}(r) d \mathbf{r}-\frac{\rho_{+} Z_{-} e \mu}{\sqrt{3}} \int h_{010}^{-d}(r) d \mathbf{r} \\
& +\frac{\rho_{d}}{2} \sum_{l_{1} l_{2} m} \int h_{l_{1} l_{2} m}^{d d}(r) \gamma_{l_{1} l_{2} m}^{d d}(r) d \mathbf{r}-\frac{\rho_{d}}{2} \sum_{l_{1} l_{2} m} \int h_{l_{1} l_{2} m}^{d d^{\prime}}(r) \gamma_{l_{1} l_{2} m}^{d d^{\prime}}(r) d \mathbf{r}
\end{aligned}
$$

where $\gamma_{l_{1} l_{2} m}^{\alpha \beta}=h_{l_{1} l_{2} m}^{\alpha \beta}-c_{l_{1} l_{2} m}^{\alpha \beta}$, and $\gamma^{d \pm *}=\gamma^{d \pm}+\beta u^{d \pm}$, so that one deals with short-range functions and the long-range behavior of the ion-dipole potential can be treated explicitly.

\section{Isothermal compressibility}

As seen in Ref. 14, this quantity is a response function, and consequently it will solely depend on the connected part of the fluid-fluid correlation function. Here, as in Ref. 14 one gets

$$
\beta\left(\frac{\beta P}{\rho_{d}}\right)_{T}=1-4 \pi \rho_{d} \int d r r^{2} c_{000}^{c}(r) .
$$

\section{B. Dielectric constant}

Applying linear response theory to the replicated system, it is possible to calculate the dielectric susceptibility of the partly quenched system in the limit $s \rightarrow 0$ (where $s$ is the number of replicas). Since the matrix does not respond to an external field, the expression is identical to the one obtained for neutral matrices ${ }^{14}$

$$
\frac{(\epsilon-1)(2 \epsilon+1)}{3 \epsilon}=\frac{4 \pi}{3} \rho_{d} \mu^{* 2}\left[1+\frac{\rho_{d}}{3} \widetilde{h}_{c}^{110}(0)\right],
$$

where $\widetilde{h}_{c}^{110}=\widetilde{h}_{110}^{c}-2 \widetilde{h}_{111}^{c}$, and the reduced dipole moment is defined by $\mu^{* 2}=\beta \mu^{2} / \sigma_{d d}^{3}$. It can be remarked that Eq. (10) is similar to the expression derived by Klapp and Patey ${ }^{20}$ for positionally frozen dipolar fluids once the local freezing order parameters are set to zero.

We note that for the dipolar fluid confined in a neutral matrix one recovers the behavior of the bulk dipolar fluid, since the relevant angular component of the blocking correlation vanishes. This will no longer be the case when charges are present, since the convolution of the nonvanishing angular components of the matrix-fluid interaction leads to a nonvanishing $h_{d d^{\prime}}^{110}$ and hence $h_{c}^{110} \neq h_{d d}^{110}$.

\section{LONG-RANGE BEHAVIOR OF THE CORRELATION FUNCTIONS}

In the absence of a matrix, the long-range behavior of the dipole-dipole correlation function $h_{d d}^{112}=h_{110}^{d d}+h_{111}^{d d}$ in the long wavelength limit is given by ${ }^{21}$ 
TABLE I. Thermodynamic and dielectric properties of a dipolar fluid adsorbed in a charged matrix obtained from a low temperature quench $\left(\beta_{0} e^{2} / \sigma=1\right)$ compared with a corresponding equilibrated ion-dipole mixture in the HNC approximation (denoted by Eq). $\beta \mu_{1}$ is the total chemical potential.

\begin{tabular}{|c|c|c|c|c|c|c|c|c|c|c|c|c|c|}
\hline \multirow[b]{2}{*}{$\rho_{0} \sigma^{3}$} & \multirow[b]{2}{*}{$\rho_{d} \sigma^{3}$} & \multicolumn{3}{|c|}{$\beta \mu_{1}$} & \multicolumn{3}{|c|}{$\beta U_{10}^{\mathrm{ex}} / V$} & \multicolumn{3}{|c|}{$\beta U_{11}^{\mathrm{ex}} / V$} & \multicolumn{3}{|c|}{$\varepsilon$} \\
\hline & & GCMC & ROZ & Eq. & GCMC & $\mathrm{ROZ}$ & Eq. & GCMC & $\mathrm{ROZ}$ & Eq. & GCMC & $\mathrm{ROZ}$ & Eq. \\
\hline 0.2 & 0.095 & -2.000 & -2.136 & -1.307 & -0.031 & -0.032 & -0.034 & -0.125 & -0.117 & -0.120 & $2.6 \pm 0.2$ & 2.581 & 2.639 \\
\hline 0.2 & 0.441 & 1.874 & 2.535 & 2.922 & -0.072 & -0.088 & -0.092 & -1.397 & -1.361 & -1.360 & $16 \pm 2$ & 15.718 & 16.697 \\
\hline 0.2 & 0.647 & 7.533 & 9.711 & $\ldots$ & -0.082 & -0.112 & $\ldots$ & -2.522 & -2.486 & $\ldots$ & $37 \pm 5$ & 49.239 & $\ldots$ \\
\hline 0.4 & 0.113 & 0.301 & 0.549 & 1.448 & -0.061 & -0.063 & -0.067 & -0.160 & -0.147 & -0.160 & $3 \pm 0.5$ & 2.827 & 3.047 \\
\hline 0.4 & 0.343 & 5.491 & 6.712 & 6.295 & -0.111 & -0.138 & -0.145 & -0.967 & -0.930 & -0.982 & $10 \pm 2$ & 9.597 & 15.100 \\
\hline 0.4 & 0.393 & 7.840 & 8.907 & $\ldots$ & -0.118 & $\cdots 0.151$ & $\ldots$ & -1.203 & -1.156 & $\ldots$ & $11 \pm 2$ & 11.637 & $\ldots$ \\
\hline
\end{tabular}

$$
\lim _{k \rightarrow 0} \widetilde{h}_{d d}^{112}(k)=-\frac{4 \pi}{3 \epsilon} \beta \mu^{\mathrm{eff}^{2}}
$$

with

$$
\mu^{\mathrm{eff}}=\frac{\epsilon-1}{3 y} \mu
$$

and $y=4 \pi \beta \rho_{d} \mu^{2} / 9$. This translates in $r$ space into

$$
\lim _{r \rightarrow \infty} h^{112}(r)=\frac{\beta \mu^{\text {eff }^{2}}}{\epsilon r^{3}} .
$$

In the equilibrated ion-dipole mixture, due to screening effects one gets, however, ${ }^{21} \lim _{k \rightarrow 0} \widetilde{h}_{d d}^{112}(k)=0$. From Eqs. (4a) to $(4 \mathrm{e})$ it can be shown that quenching the ionic positions leads back to the behavior of Eqs. (11) and (12) for the connected part of the dipole-dipole correlation function, which plays the role of the fluid-fluid correlation in partly quenched systems. That is, here we obtain

$$
\lim _{r \rightarrow \infty} h_{c}^{112}(r)=\frac{\beta \mu^{\mathrm{eff}^{2}}}{\epsilon r^{3}}
$$

Equations (13) and (10) should lead to a consistent value of the dielectric constant, and this is indeed the case in our calculations.

As to the $h^{110}$ component, its long wavelength behavior in the bulk dipolar fluid is connected with the dielectric function by an expression identical to Eq. (10), whereas in the equilibrated ion-dipole mixture one finds ${ }^{21}$

$$
\frac{\epsilon-1}{3 y}=1+\frac{\rho_{d}}{3} \widetilde{h}_{d d}^{110}(0)
$$

As we have seen, quenching the ions transforms Eq. (14) into Eq. (10). It can also be shown that the long-range behavior of one of the components of $h_{d d}^{110}(r), h_{110}^{d d}(r)$, gets Coulombic due to the quenching, namely

$$
\lim _{k \rightarrow 0} \widetilde{h}_{110}^{d d}(k)=\frac{4 \pi \beta \mu^{2} e^{2}\left(Z_{-}^{2} \rho_{-}\left(1+\rho_{-} \widetilde{h}_{--}(0)\right)+Z_{+}^{2} \rho_{+}\left(1+\rho_{+} \widetilde{h}_{++}(0)\right)+2 \rho_{-} \rho_{+} Z_{-} Z_{+} \widetilde{h}_{-+}(0)\right)}{3 k^{2}\left(1-\widetilde{c}_{110}^{c}(0)\right)} .
$$

The results found by Holovko and Polishchuk ${ }^{22}$ for an iondipole system adsorbed in ion-dipolar matrices are consistent with this behavior. This Coulombic tail is particularly relevant since it has to be properly dealt with in the numerical procedure to solve the ROZ equations. In this respect, an adequate treatment of the long-range behavior of the correlation functions can be constructed following the prescriptions of Chen and Forstmann ${ }^{23}$ for the ion-dipole mixture.

\section{SIMULATION DETAILS}

In the simulations the solid porous medium is considered as an ensemble of frozen equilibrium configurations of a system of charged hard spheres. These configurations are randomly chosen among those obtained in canonical ensemble simulations of $N_{0}$ hard spheres $\left(N_{0} / 2\right.$ charged positively and $N_{0} / 2$ charged negatively) at density $\rho_{0} \sigma_{00}^{3}$. The equilibrium state of the fluid of dipolar hard spheres adsorbed in each of the selected matrix configurations is determined, at given chemical potential and temperature, by simulation in the grand canonical ensemble.

The details of realization of such simulations have been described in the literature, in particular in Refs. 2, 7, and 24. Charge-charge, charge-dipole, and dipole-dipole interactions have been evaluated by an Ewald summation in the canonical and grand canonical simulations, the volume $V$ containing the matrix and the fluid particles being a cube with periodic boundary conditions. In the Ewald sums, the continuous dielectric medium supposed to surround the periodically repeated simulation cell, is taken to be a conducting medium and thus of infinite dielectric constant (tin foil boundary conditions).

Correlation functions and thermodynamic properties have been obtained by averaging over $16-20 \times 10^{7}$ elementary GCMC moves (displacement, insertion, and deletion of 
TABLE II. Thermodynamic and dielectric properties of a dipolar fluid adsorbed in a charged matrix obtained from a high temperature quench $\left(\beta_{0} e^{2} / \sigma\right.$ $=0.005) . \quad \beta \mu_{1}$ is the total chemical potential.

\begin{tabular}{|c|c|c|c|c|c|c|c|c|c|}
\hline \multirow[b]{2}{*}{$\rho_{0} \sigma^{3}$} & \multirow[b]{2}{*}{$\rho_{d} \sigma^{3}$} & \multicolumn{2}{|c|}{$\beta \mu_{1}$} & \multicolumn{2}{|c|}{$\beta U_{10}^{\mathrm{ex}} / V$} & \multicolumn{2}{|c|}{$\beta U_{11}^{\mathrm{ex}} / V$} & \multicolumn{2}{|c|}{$\varepsilon$} \\
\hline & & GCMC & $\mathrm{ROZ}$ & GCMC & $\mathrm{ROZ}$ & GCMC & $\mathrm{ROZ}$ & GCMC & $\mathrm{ROZ}$ \\
\hline 0.2 & 0.081 & -2.3165 & $\ldots$ & -0.061 & $\ldots$ & -0.085 & $\ldots$ & $2.3 \pm 0.1$ & $\ldots$ \\
\hline 0.2 & 0.314 & 0.0839 & 0.1810 & -0.116 & -0.123 & -0.804 & -0.797 & $9.6 \pm 0.5$ & 8.759 \\
\hline 0.2 & 0.654 & 7.8600 & 10.034 & -0.149 & -0.171 & -2.522 & -2.512 & $37 \pm 5$ & 48.208 \\
\hline 0.4 & 0.117 & 0.0854 & 0.508 & -0.161 & -0.146 & -0.132 & -0.146 & $3 \pm 0.5$ & 2.630 \\
\hline 0.4 & 0.258 & 2.9626 & 3.753 & -0.217 & -0.229 & -0.546 & -0.549 & $7 \pm 0.8$ & 5.784 \\
\hline 0.4 & 0.386 & 7.3104 & 8.459 & -0.254 & -0.278 & -1.080 & -1.068 & $11 \pm 2$ & 10.025 \\
\hline
\end{tabular}

a dipolar sphere) for given matrix configuration (thermal average of fluid state) and over six different matrix configurations (average over disorder). The thermodynamic states considered in this work (summarized in Tables I and II) correspond to homogeneous states, unambiguously identified by the existence of a unique peak in the distribution of number of dipolar spheres, $N_{d}$, evaluated in the GCMC simulations. As shown in Refs. 2 and 24, for such homogeneous states, a limited number of matrix configurations is required to obtain a reliable average over disorder.

The GCMC values of $\rho_{d} \sigma_{d d}^{3}, \beta U_{10}^{\mathrm{ex}} / V$, and $\beta U_{11}^{\mathrm{ex}} / V$ given in Table I have statistical errors of the order of $1 \%-$ $2 \%$; similar error affects the correlation functions $h_{d d}^{000}$ or $h_{d d}^{110}$. The error on the GCMC value of $\varepsilon$ is appreciable for $\varepsilon$ larger than 10. The estimated statistical error combines the statistical error on the evaluation of $\varepsilon$ at given matrix configuration with that associated with the average over disorder.

The expression used for calculating $\varepsilon$ in the GCMC simulations is

$$
\epsilon-1=\frac{4 \pi \beta}{3 V}\left\langle\left\langle\vec{M}^{2}\right\rangle_{T}\right\rangle_{m},
$$

where $\langle\cdots\rangle_{T}$ denotes thermal average, $\langle\cdots\rangle_{m}$ average over disorder, and $\vec{M}=\sum_{i=1}^{N_{d}} \vec{\mu}_{i}$ the total dipolar moment of the system.

This expression for $\varepsilon$ is identical to that for a pure dielectric fluid in a volume with periodic boundary conditions and tin foil boundary conditions associated with a conducting medium surrounding, at a macroscopic distance, the periodically repeated simulation volume. Use of this expression is based on the remarks that the external field created by the immobile charges of the matrix does not screen the electric field of the mobile dipoles, as would be the case in an electrolyte solution, and that the continuous conducting medium annuls the effect of the field created by the polarization charges. It should be noted, however, that a formal proof of Eq. (16), similar to the one developed for pure dielectric fluids or electrolyte solutions, as obtained, for instance, in Ref. 25 seems not yet to have been established.

The blocking functions $h_{d d^{\prime}}^{000}(r)$ and $h_{d d^{\prime}}^{110}(r)$ have been calculated according to the procedure described in Refs. 14 and 24 which consists in paving the simulation cell with $n^{3}$ $=64^{3}$ subvolumes, thus allowing one to evaluate $h_{d d^{\prime}}^{000}$ and $h_{d d^{\prime}}^{110}$ by means of a three-dimensional fast Fourier transform.

\section{RESULTS AND CONCLUSIONS}

In this work we have focused on the simplest case where matrix and fluid particles have equal sizes, hence $\sigma_{00}=\sigma_{0 d}$ $=\sigma_{d d}=\sigma$. The reduced dipole-dipole and charge-dipole couplings have been set to $\mu^{* 2}=2.75$ and $\beta \mu e / \sigma^{2}=1.658$ for the two different matrix configurations considered, corresponding to ionic particle positions quenched at reduced inverse temperatures $\beta_{0} e / \sigma=1$ and 0.005 , respectively. The latter case implies that charges in this system are randomly distributed. In both cases we have studied two different matrix densities $\rho_{0} \sigma^{3}=\left(\rho_{+}+\rho_{-}\right) \sigma^{3}=0.2$ and 0.4 , and various fluid densities. The thermodynamic states under consideration are collected in Tables I and II.

The solution of the ROZ equations was carried out on a discretized mesh of 8192 points with a grid size $0.01 \sigma$. The same conditions were used to solve the HNC equation for the corresponding equilibrated mixtures.

In Fig. 1 and Tables I and II we present the results for the internal energy and chemical potential for the two model matrices under consideration. Additionally in Table I we have included the HNC results for the corresponding equilibrated mixtures of ions and dipoles. The ROZ results agree

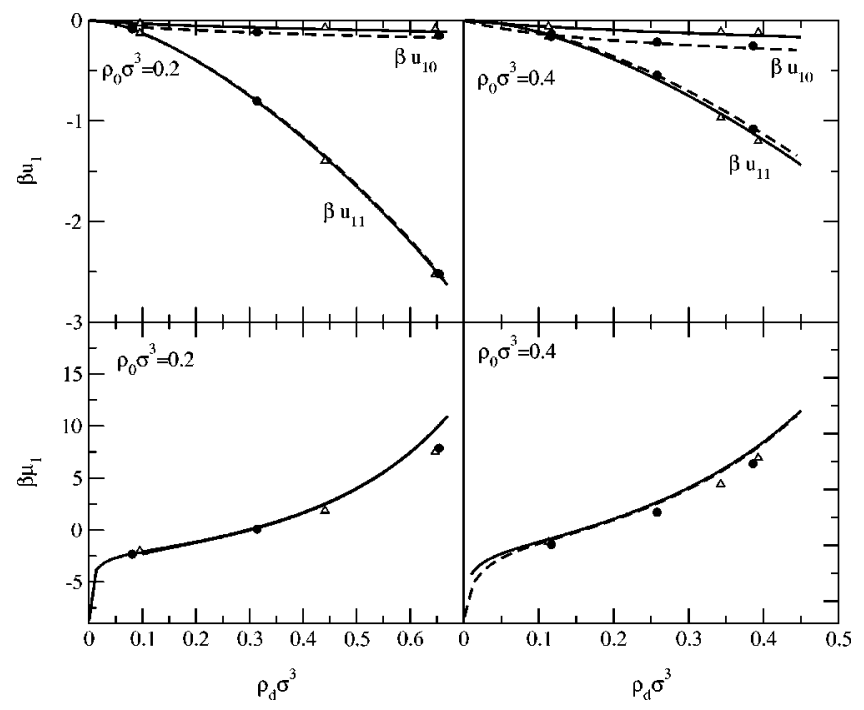

FIG. 1. Excess internal energy [split in ion-dipole $\left(\beta u_{10}=\beta U_{10}^{\mathrm{ex}} / V\right)$ and dipole-dipole $\left(\beta u_{11}=\beta U_{11}^{\mathrm{ex}} / V\right)$ contributions $]$ and chemical potential for a dipolar fluid inclusion in ionic matrices quenched at low temperature $\beta_{0} e^{2} / \sigma=1$ (solid line and closed triangles) and high temperature $\beta_{0} e^{2} / \sigma$ $=0.005$ (dotted line and closed circles). 
reasonably well with the simulation, and one observes some minor differences when comparing the behavior of the dipolar fluids within the matrix quenched at the same temperature and inside the high temperature (i.e., with randomly distributed charges) matrix. In the latter case the charge-dipole contribution to the internal energy seems substantially larger when compared with the low temperature quench situation. This is probably due to the fact that in the low temperature quench case, the matrix charge and particle position distributions correspond to that of a primitive model electrolyte, and consequently exhibit a certain degree of pairing and charge screening. As a result, the dipoles will interact with positive and negative charges in many cases distributed in pairs, by which a significant portion of the interaction energy will cancel. This partial cancellation will certainly not take place when the charges are randomly distributed. This has important consequences in the convergence properties of the integral equation for low matrix and low fluid densities for the high temperature matrix case, to the point that the integral equation breaks down before reaching the lowest density state presented in Table II. Aside from this, a comparison with the equilibrated mixture $\mathrm{HNC}$ results shows that we are now dealing with a situation in which the partial quenching alters considerably the behavior of the chemical potential, and to a much lesser extent the internal energy. As the dipolar density is increased the HNC equation breaks down. We will see later on that this is very likely due to a demixing transition.

As to the dielectric constant, this quantity is plotted in Figs. 2 and 3 for the two types of system. The large discrepancies with GCMC at high density might be due in part to the HNC closure, but one must also bear in mind that the GCMC results for a quantity like the dielectric constant at these high densities are plagued with appreciable uncertainties. The strange behavior of the dielectric constant for the equilibrated mixture can be correlated to an incipient demixing transition. On the other hand the dielectric properties of the dipolar fluid do not seem to be essentially affected by the distribution of charges in the matrix. The comparison with the uncharged matrix results indicates that the presence of charges (whatever their distribution might be) lowers the response of the dipoles to an external field, i.e., lowers the dielectric constant. This can easily be understood, since the local field formed by the matrix charges will somehow tend to impede the reorientation of the dipoles as compared with the neutral matrix.

In order to analyze the convergence difficulties of the $\mathrm{HNC}$ for the ion-dipole mixtures we have resorted to the stability analysis of Chen and Forstmann. ${ }^{26}$ In Ref. 23 these authors generalized their treatment to ion-dipole mixtures of equal size. Their analysis is based on the study of the stability of the grand potential functional. The fluctuations in this quantity for the present case can be cast in the form ${ }^{23}$

$$
\delta \Omega=\frac{1}{2 \beta V}\left(\begin{array}{ll}
\delta \tilde{\rho}(0) & \delta \tilde{c}(0))
\end{array}\right)\left(\begin{array}{ll}
M_{\rho \rho} & M_{\rho c} \\
M_{c \rho} & M_{c c}
\end{array}\right)\left(\begin{array}{c}
\delta \tilde{\rho}(0) \\
\delta \tilde{c}(0)
\end{array}\right),
$$

where the density and concentration fluctuations are

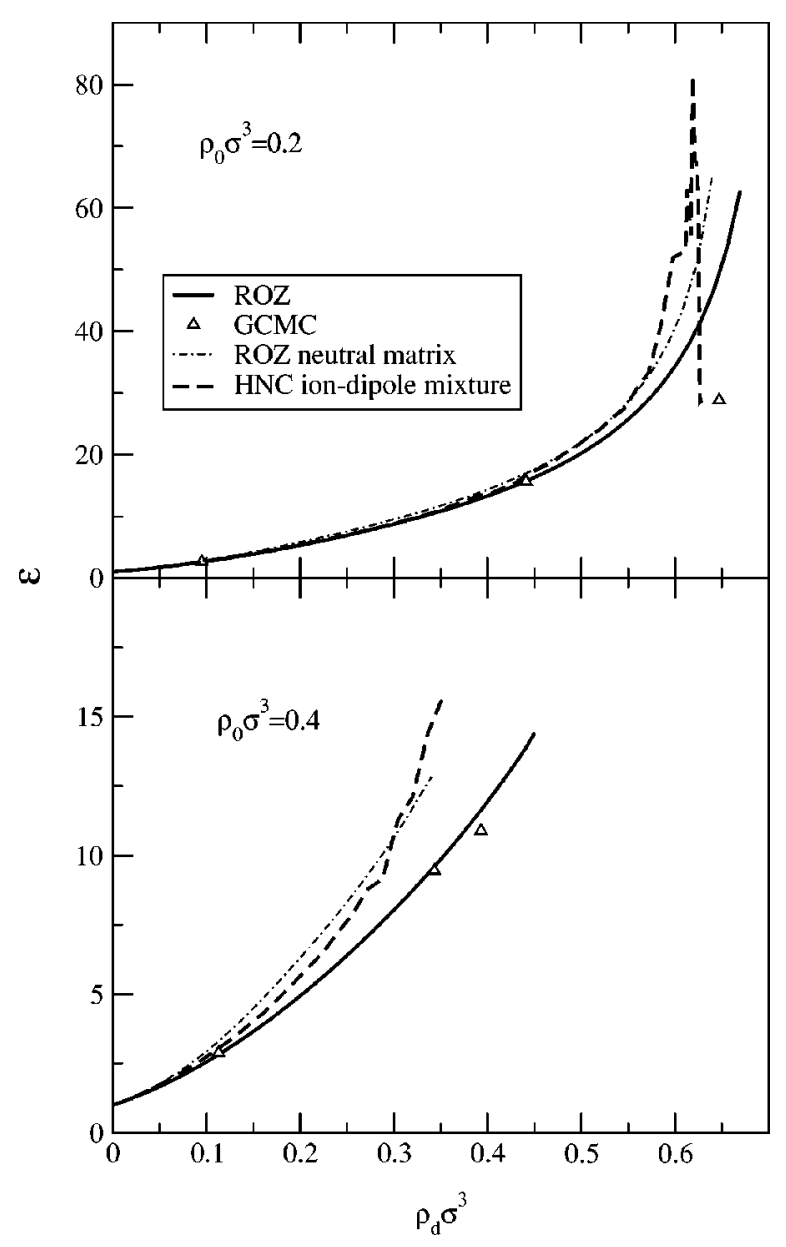

FIG. 2. Dielectric constant of the dipolar fluid embedded in a charged matrix quenched at low temperature $\beta_{0} e^{2} / \sigma=1$. ROZ vs GCMC results. HNC results for the corresponding equilibrated mixture and $\mathrm{ROZ}$ results for an equivalent system with a neutral (hard sphere) matrix are included for comparison.

$$
\begin{aligned}
& \delta \tilde{\rho}(0)=\rho^{-1 / 2}\left(\delta \tilde{\rho}_{0}(0)+\delta \tilde{\rho}_{d}(0)\right), \\
& \delta c(0)=\rho^{-3 / 2}\left(c_{0} c_{d}\right)^{-1 / 2}\left(\rho_{d} \delta \tilde{\rho}_{0}(0)-\rho_{0} \delta \tilde{\rho}_{d}(0)\right)
\end{aligned}
$$

with $\rho=\rho_{0}+\rho_{d}, c_{i}=\rho_{i} / \rho$, and $\delta \tilde{\rho}_{d}(0)=\sqrt{4 \pi} \delta \tilde{\rho}_{d}^{00}(0)$. This latter quantity is the Fourier transform of the radial average of the one particle dipole density fluctuation. The coefficients of the symmetric $\mathbf{M}$ matrix are ${ }^{23}$

$$
\begin{aligned}
& M_{\rho \rho}=1-\rho\left[c_{0}^{2} \tilde{c}_{00}(0)+c_{d}^{2} \tilde{c}_{000}^{d d}(0)+2 c_{0} c_{d} \tilde{c}_{000}^{+d}(0)\right], \\
& M_{c c}=1-\rho c_{0} c_{d}\left[\tilde{c}_{00}(0)+\tilde{c}_{000}^{d d}(0)-\tilde{c}_{000}^{+d}(0)\right], \\
& M_{\rho c}=\rho \sqrt{c_{0} c_{d}}\left[c_{d} \tilde{c}_{000}^{d d}(0)-c_{0} \tilde{c}_{00}(0)-\left(c_{d}-c_{0}\right) \tilde{c}_{000}^{+d}(0)\right]
\end{aligned}
$$

with $\tilde{c}_{00}=\left(\tilde{c}_{++}+\tilde{c}_{+-}\right) / 2$. One can then determine the eigenvalues of the matrix $M$, which are given by

$$
\lambda_{1,2}=\frac{M_{\rho \rho}+M_{c c} \mp \sqrt{\left(M_{\rho \rho}-M_{c c}\right)^{2}+4 M_{\rho c}^{2}}}{2} .
$$

Now, the minimum eigenvalue $\lambda_{\min }=\min \left(\lambda_{1}, \lambda_{2}\right)$ decides the stability of the phase. If $\lambda_{\min } \rightarrow 0$ the phase will be unstable. Depending on the components of the eigenvector we will have a demixing transition or a gas-liquid instability. 


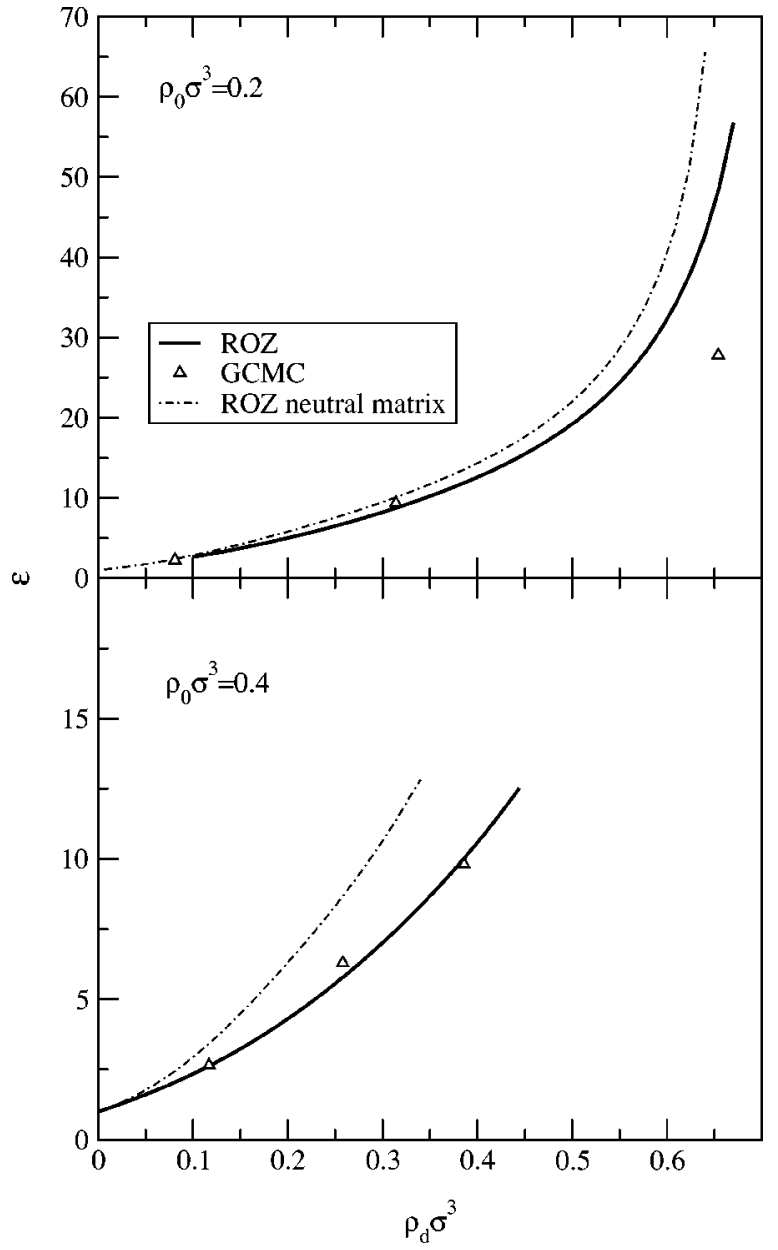

FIG. 3. Dielectric constant of the dipolar fluid embedded in a charged matrix quenched at high temperature $\beta_{0} e^{2} / \sigma=0.005$. ROZ vs GCMC results. $\mathrm{ROZ}$ results for an equivalent system with a neutral (hard sphere) matrix are included for comparison.

Note that a gas-liquid instability will also be signaled by a divergence in the isothermal compressibility. These two quantities, obtained in the HNC approximation, are plotted in Fig. 4 for the equilibrated ion-dipole mixtures of interest in this paper. We see that the minimum eigenvalue shows an evident decrease as the dipole density increases whereas the isothermal compressibility is monotonously decreasing. This is a clear indication of the tendency to demix. The oscillations observed in the $\lambda_{\min }$ curve at high dipole densities are due to numerical instabilities in the solution of the HNC equation as the correlation functions become more and more long ranged.

Regarding the microscopic structure of the partly quenched system, perhaps the most relevant quantity is the fluid-matrix correlation, in particular the first angular coefficient, $h_{100}^{d \pm}$. This quantity is plotted for a high density state in Fig. 5. Other correlation functions behave much in the same way as in bulk fluids and mixtures and for the sake of brevity are not presented here. We observe in Fig. 5 that the correlation obtained by simulation for the high temperature matrix is considerably longer ranged. The $\mathrm{ROZ}$ equations reproduce rather well its behavior except at contact, where the correlations are much overestimated. This effect is most

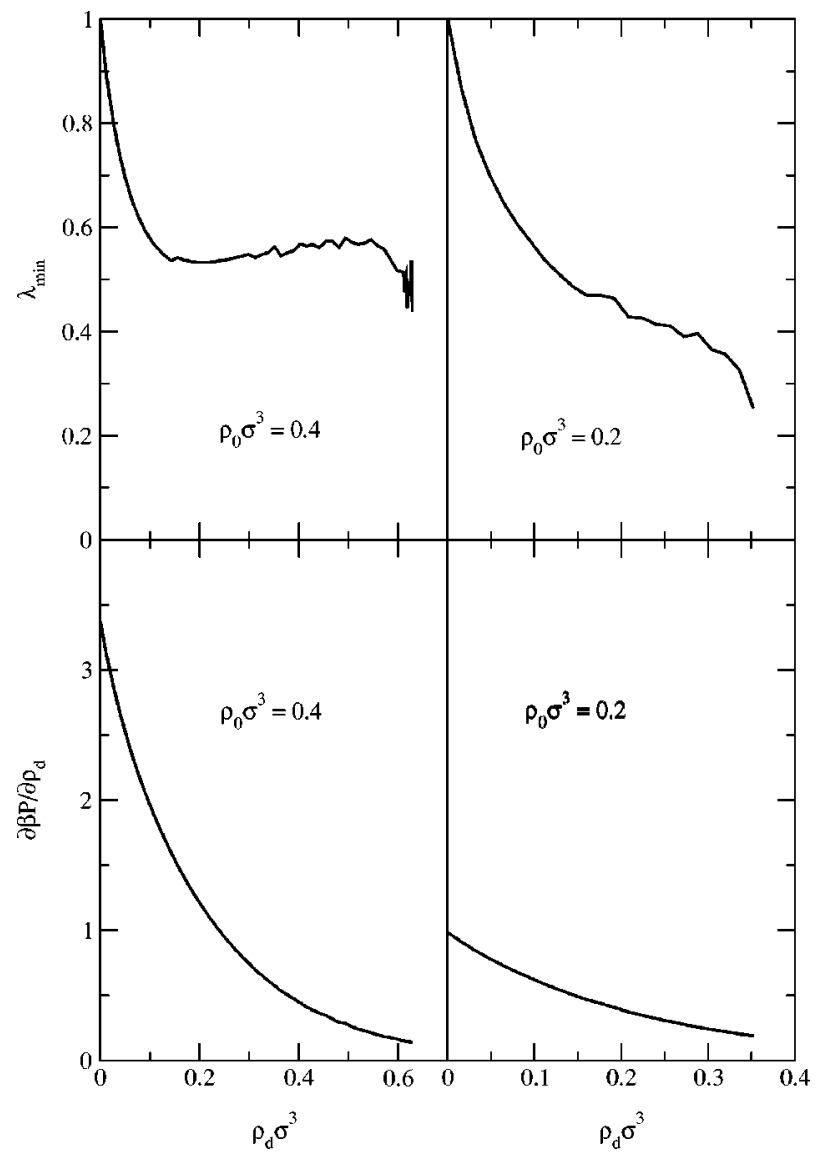

FIG. 4. Minimum eigenvalue of the stability matrix [see Eq. (20)] and isothermal compressibility for equilibrated mixtures of charged and dipolar hard spheres.

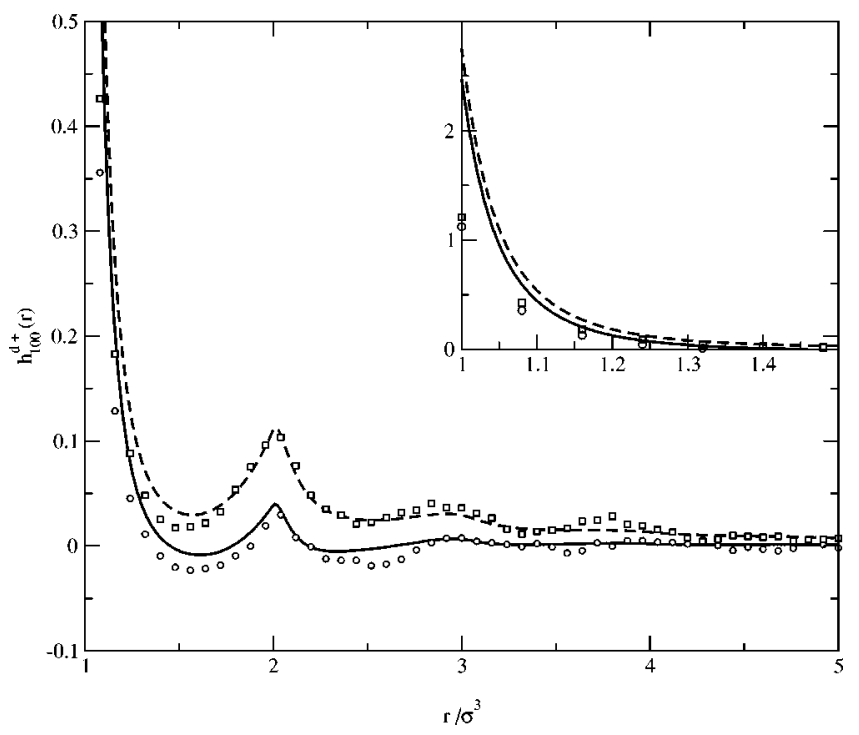

FIG. 5. Leading angular coefficient of the ion-dipole correlation function for the two matrix topologies considered calculated by means of the ROZ integral equation (lines) and from GCMC simulation (symbols). The states under consideration correspond to $\rho_{0} \sigma^{3}=0.4, \rho_{d} \sigma^{3}=0.386$ for the high temperature matrix (solid line and circles) and $\rho_{d} \sigma^{3}=0.393$ for the low temperature matrix (dotted line and squares). 


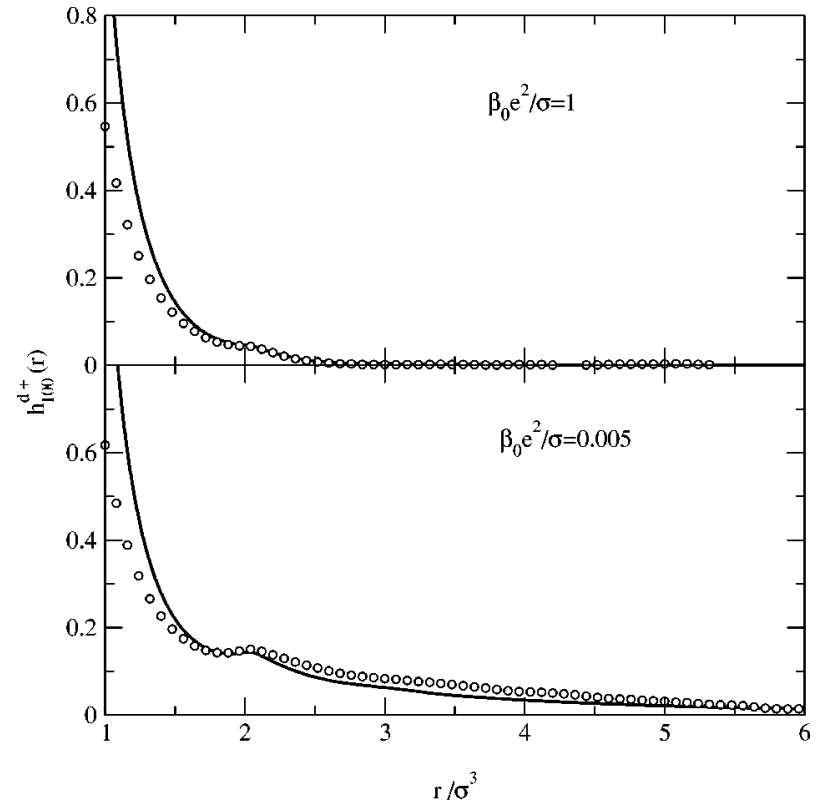

FIG. 6. Leading angular coefficient of the ion-dipole correlation function for the two matrix topologies for low density states $\left(\rho_{0} \sigma^{3}=0.2, \rho_{d} \sigma^{3}\right.$ $=0.095$ - upper graph—and $\rho_{d} \sigma^{3}=0.081$-lower graph) by means of the $\mathrm{ROZ}$ integral equation (lines) and from GCMC simulation (symbols). In the case of the high temperature matrix quench (lower graph) the ROZ results correspond to the lowest attainable dipole density, $\rho_{d} \sigma^{3}=0.089$.

likely due to the HNC closure used in the ROZ equations.

Now if we lower the density the difference between the matrix-fluid correlations for the two types of matrix become even more significant. This is illustrated in Fig. 6. We observe there that for the high temperature matrix the angular correlations become appreciably long ranged (up to five diameters). Actually, in this case the theory could not reach the simulation density due to convergence problems in the ROZ equations. The contact values are similar for the low temperature matrix but the correlations die out more rapidly, and this time there was no problem to lower the density in the ROZ equations. This implies there could be a correlation between the long range of $h_{100}^{d \pm}$ and the lack of convergence of the integral equation. If we now examine the dipoledipole coefficient $h_{d d}^{110}$, plotted in Fig. 7, we see that there is a clear indication that in both cases the dipoles exhibit a dominant head-to-tail alignment, but in the case of the high temperature matrix it includes to some extent up to second neighbors. At high matrix densities we did not encounter convergence difficulties for the two matrix configurations. It seems rather clear that at low $\rho_{0}$ the charged matrix particles induce a head-to-tail alignment of the dipoles around them. This alignment is somewhat impeded in the low temperature matrix, since in this case ions of different signs are clustered together (i.e., screen each other) and distort the ordering of the dipoles-see Fig. 8 for a comparison of the unlike pair distribution functions. All this implies that the local density of ions is higher in the case of the low temperature matrix. Obviously the same effect can be achieved by a simple increase of the matrix density, and this explains why the correlations in Fig. 5 are not so long ranged as in Fig. 6 and no convergence difficulties appear in the ROZ equations at

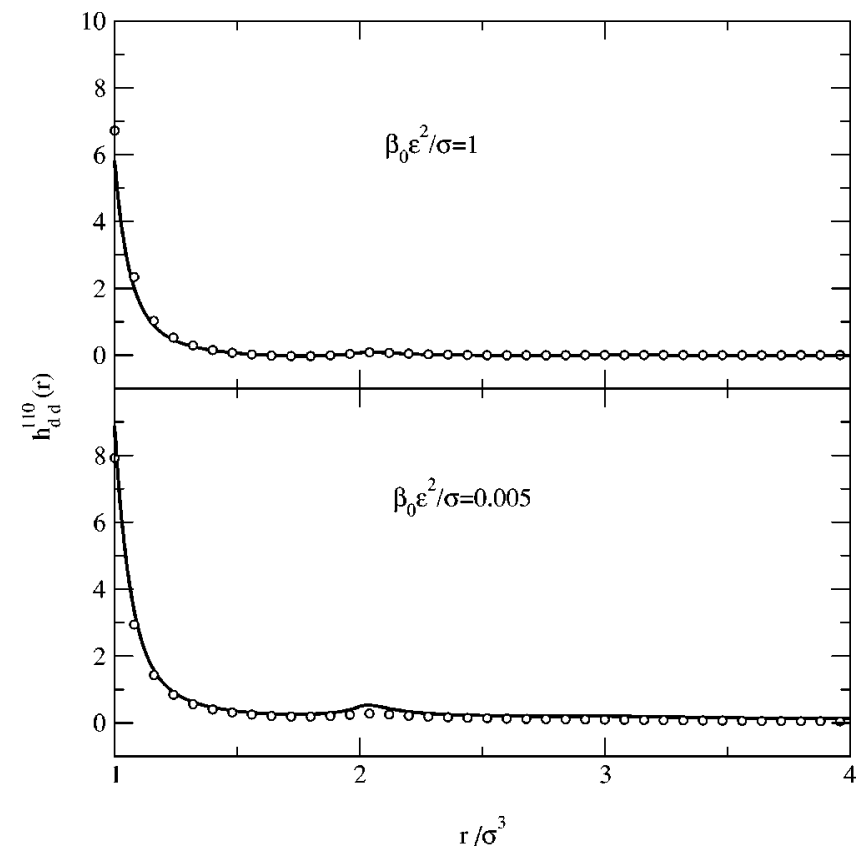

FIG. 7. Dipole-dipole $h_{d d}^{110}$ component of the total correlation function for the two matrix topologies for low density states $\left(\rho_{0} \sigma^{3}=0.2\right.$, $\rho_{d} \sigma^{3}=0.095$ - upper graph - and $\rho_{d} \sigma^{3}=0.081$-lower graph) by means of the ROZ integral equation (lines) and from GCMC simulation (symbols). In the case of the high temperature matrix quench (lower graph) the ROZ results correspond to the lowest attainable dipole density, $\rho_{d} \sigma^{3}=0.089$.

$\rho_{0} \sigma^{3}=0.4$. The break down of the HNC equation for bulk dipolar systems in the low density regime is a well-known feature, and has been attributed to the inability of the equations to account for clustering effects beyond pairing. It is clear that the lack of screening in the high temperature matrix enhances dipole association, and one can easily understand why the ROZ equations break down here in the low density regime as well.

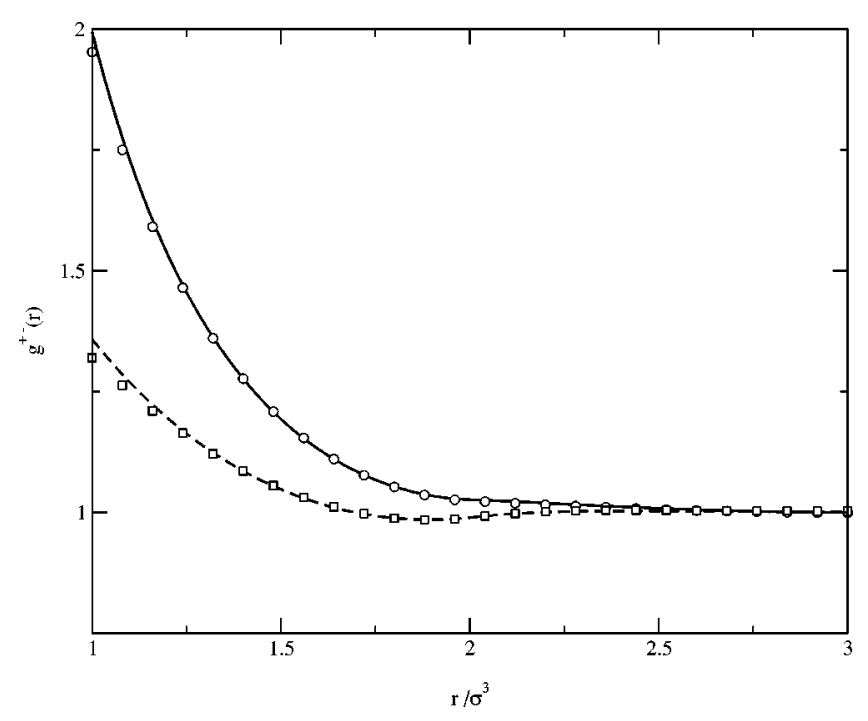

FIG. 8. Matrix-matrix unlike distribution function for $\rho_{0} \sigma^{3}=0.2$ for the low temperature (solid line and circles) and high temperature (dotted line and squares) matrix. In the latter case like-like and unlike correlations are identical to the uncharged hard sphere pair distribution function. 


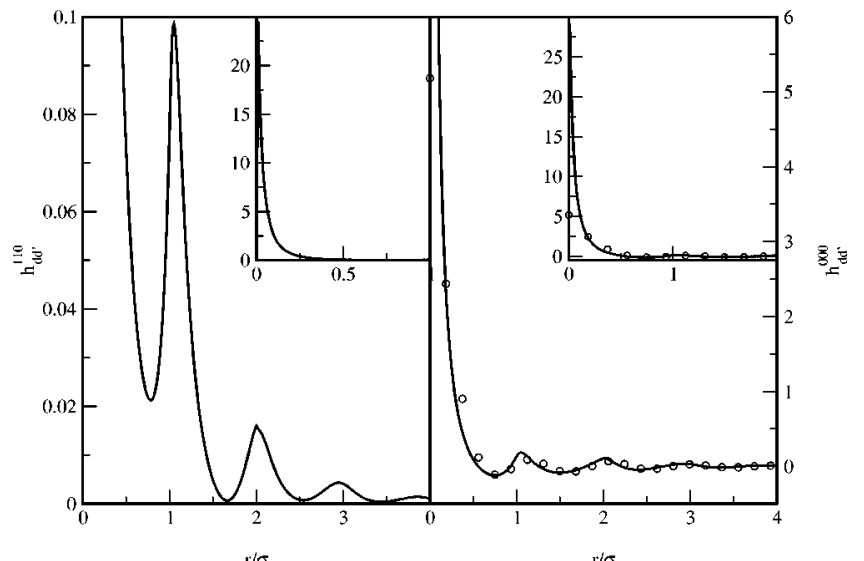

FIG. 9. Blocking functions for a dipolar fluid adsorbed in a charged hard sphere matrix $\left(\beta_{0} e^{2} / \sigma=1\right)$ at $\rho_{0} \sigma^{3}=0.2$ and $\rho_{d} \sigma^{3}=0.441$. The angular component $h_{d d^{\prime}}^{110}$ is plotted on the left-hand side and the radial term $h_{d d^{\prime}}^{000}$ on the right-hand side. Lines represent HNC-ROZ results and circles GCMC simulation.

Figure 9 presents a comparison between the theoretical blocking functions $h_{d d^{\prime}}^{000}(r)$ and $h_{d d^{\prime}}^{110}(r)$ and those obtained by simulation for the state $\rho_{0} \sigma^{3}=0.2, \rho_{d} \sigma^{3}=0.441$ and total chemical potential $\beta \mu_{1}=1.874$ (cf. Table I) in the case of the low temperature matrix $\beta_{0} e^{2} / \sigma=1$. They are typical of those obtained for other state points. The agreement between $h_{d d^{\prime}}^{000}(r)$ obtained by simulation and ROZ equation is qualitatively and quantitatively satisfactory for $r>0.3 \sigma$. A clear quantitative disagreement exists for $r<0.3 \sigma$, which as discussed in Ref. 24 is essentially due to the approximation entailed by the choice of the closure of the ROZ equations.

The ROZ correlation function $h_{d d^{\prime}}^{110}(r)$ plotted in Fig. 9 suggests that the quenched charges induce a local polarization in the adsorbed polar fluid for each matrix configuration. For instance, $h_{d d^{\prime}}^{110}(0)$, which is proportional to $\left\langle(\vec{M}(0)\rangle_{T} \cdot\langle\vec{M}(0)\rangle_{T}\right\rangle_{m}-\left\langle\langle\vec{M}(0)\rangle_{T}\right\rangle_{m}^{2}$, is positive and has a large value of 20.0 for the state represented in Fig. 9. This result is in qualitative disagreement with the simulation data because, for all the states considered in Table I, and all values of $r,\left\langle\langle\vec{M}(0)\rangle_{T} \cdot\langle\vec{M}(r)\rangle_{T}\right\rangle_{m}-\left\langle\langle\vec{M}(0)\rangle_{T}\right\rangle_{m}^{2}$ is zero within the statistical uncertainties. This observation is compatible with $\langle\vec{M}(r)\rangle_{T} \simeq 0$ indicating at least that the charge-dipole coupling is insufficient to locally polarize the dielectric fluid with the values of $Z$ and $\mu$ considered. We lack a satisfactory explanation for this discrepancy.

In summary, we have presented a study of the behavior of a dipolar fluid inclusion in charged matrices obtained by quenching the charged hard sphere positions at the same temperature than the dipole fluid and at a temperature two hundred times higher. The latter case implies a random distribution of charges with no screening. We have observed that the two systems present substantial differences with respect to the fully equilibrated mixtures, being however rather similar to each other. One exception to this similarity is found in the low density microscopic structure, in which the lack of screening in the high temperature matrix enhances dipole-dipole association to the point that the ROZ equations with the HNC closure break down for rather low dipole moments. In the domain where solutions can be found, the results provided by the ROZ equations are generally in fair agreement with the simulations.

\section{ACKNOWLEDGMENTS}

Two of the authors (E.L. and C.M.) acknowledge support from the Dirección General de Investigación Científica y Técnica under Grant No. BFM2001-1017-C03-01.

${ }^{1}$ W.G. Madden and E.D. Glandt, J. Stat. Phys. 51, 537 (1988).

${ }^{2}$ M. Alvarez, D. Levesque, and J.J. Weis, Phys. Rev. E 60, 5495 (1999).

${ }^{3}$ M.J. Fernaud, E. Lomba, and J.J. Weis, Phys. Rev. E 64, 051501 (2001).

${ }^{4}$ A. Kovalenko and F. Hirata, J. Chem. Phys. 115, 8620 (2001).

${ }^{5}$ A. Trokhymchuk, O. Pizio, M. Holovko, and S. Sokolowski, J. Phys. Chem. 100, 17004 (1996).

${ }^{6}$ P. Padilla, O. Pizio, A. Trokhymchuk, and C. Vega, J. Phys. Chem. 102, 3012 (1998).

${ }^{7}$ E. Schöll-Paschinger, D. Levesque, J.J. Weis, and G. Kahl, Phys. Rev. E 64, 011502 (2001).

${ }^{8}$ E. Lomba, J. Given, G. Stell, and J.J. Weis, Phys. Rev. E 48, 233 (1993).

${ }^{9}$ P.R. van Tassel, Phys. Rev. E 60, R25 (1999).

${ }^{10}$ W. Rzysko, S. Sokolowski, and O. Pizio, J. Chem. Phys. 116, 4286 (2002).

${ }^{11}$ B. Hribar, O. Pizio, A. Trokhymchuk, and V. Vlachy, J. Chem. Phys. 107, 6335 (1997); 109, 2480 (1998).

${ }^{12}$ B. Hribar, V. Vlachy, and O. Pizio, Mol. Phys. 100, 3093 (2002).

${ }^{13}$ D.M. Ford, A.P. Thompson, and E.D. Glandt, J. Chem. Phys. 103, 1099 (1995).

${ }^{14}$ M.J. Fernaud, E. Lomba, J.J. Weis, and D. Levesque, Mol. Phys. (to be published).

${ }^{15}$ C. Spöler and S.H.L. Klapp, J. Chem. Phys. 118, 3628 (2003).

${ }^{16}$ J. Given and G. Stell, J. Chem. Phys. 97, 4573 (1992).

${ }^{17}$ J.S. Høye, E. Lomba, and G. Stell, Mol. Phys. 75, 1217 (1992).

${ }^{18}$ E. Kierlik, M.L. Rosinberg, and G. Tarjus, J. Chem. Phys. 106, 264 (1997).

${ }^{19}$ L.L. Lee, J. Chem. Phys. 97, 8606 (1992).

${ }^{20}$ S.H.L. Klapp and G.N. Patey, J. Chem. Phys. 115, 4718 (2001).

${ }^{21}$ D. Levesque, J.J. Weis, and G.N. Patey, J. Chem. Phys. 72, 1887 (1980).

${ }^{22}$ M.F. Holovko and Z.V. Polishchuk, Condens. Matter Phys. 2, 267 (1999).

${ }^{23}$ X.S. Chen and F. Forstmann, Condens. Matter Phys. 4, 679 (2001).

${ }^{24}$ A. Meroni, D. Levesque, and J.J. Weis, J. Chem. Phys. 105, 1101 (1996).

${ }^{25}$ J.M. Caillol, D. Levesque, and J.J. Weis, J. Chem. Phys. 91, 5544 (1989).

${ }^{26}$ X.S. Chen and F. Forstmann, Mol. Phys. 76, 1203 (1992). 